\title{
Common Pitfalls in the Conduct of Clinical Research
}

\author{
Michael Zlowodzki $^{a}$ Anders Jönsson $^{b}$ Mohit Bhandari $^{\mathrm{a}, \mathrm{b}}$ \\ ${ }^{a}$ Division of Orthopaedic Surgery, McMaster University, Hamilton, Canada; ${ }^{b}$ Clinical Investigation Division, \\ Trauma Care Institute, Association Internationale pour l'Ostéosynthèse Dynamique (AIOD), Nice, France
}

\author{
Key Words \\ Evidence-based medicine - Clinical research - Review • \\ Randomized trial $\cdot$ Study design
}

\begin{abstract}
Recently, paradigm shift from expert opinion towards evidence-based medicine has occurred encouraging physicians to base their treatment decisions on the best available research evidence. In the hierarchy of evidence randomized clinical trials (level 1 evidence) are considered of the highest quality (least biased) while non-randomized studies represent lower levels (levels 2-4). Several pitfalls in the design and conduct of clinical research include: lack of randomization, lack of concealment, lack of blinding, and errors in hypothesis testing (type I and II errors). A basic understanding of these principles of research will empower both investigators and readers when applying the results of research to clinical practice.
\end{abstract}

Copyright (C) 2006 S. Karger AG, Basel

\section{Introduction}

According to Sackett et al. [1] evidence-based medicine is defined as 'the conscientious, explicit, and judicious use of current best evidence in making decisions about the care of individual patients'. Recently, a para- digm shift from expert opinion towards evidence-based medicine has occurred encouraging physicians to base their treatment decisions on the best available research evidence. Consequently, ensuring the validity of published clinical research is important to ensure best practice. Both researchers and readers should be aware of common issues in the design and conduct of clinical research. Understanding basic principles of the hierarchy of evidence, errors in hypothesis testing and other basic methodological issues will empower both researchers and the consumers of research papers to apply only the highest quality evidence to their clinical practice. We present an overview of common pitfalls in clinical research.

\section{Hierarchy of Evidence}

Not all information that is published is of equal quality. To assist readers in categorizing research studies from best evidence to least helpful evidence, a 'Level of Evidence' grading system (levels 1-5) has been proposed (fig. 1) [1]. In the hierarchy of evidence randomized clinical trials (level 1 evidence) are considered of the highest quality (least biased). Some consider a statistical pooling of high quality randomized trials (i.e. called meta-analysis) as good if not better evidence than a single randomized trial. Nonrandomized comparisons like prospective cohort studies (level 2 evidence), or case-control studies (level 3 evidence) can introduce bias in their design and

\section{KARGER}

Fax +4161306 1234

E-Mail karger@karger.ch

www.karger.com
(C) 2006 S. Karger AG, Basel

1011-7571/06/0151-0001\$23.50/0

Accessible online at:

www.karger.com/mpp
Mohit Bhandari, MD

Hamilton Health Sciences - General Hospital

237 Barton Street East, 7 North Wing, Suite 727

Hamilton, Ont., L8L 2X2 (Canada)

Tel. +19055274322 ext. 44490, Fax +1905523 8781, E-Mail bhandam@mcmaster.ca 
are thus lower levels of evidence. Studies without a comparison group are considered of lower quality (level 4 evidence). Expert opinion is the weakest form of evidence (level 5 evidence). Regardless of the quality level of a study, there are numerous pitfalls that can be made during study design, study conduction and data analysis (table 1).

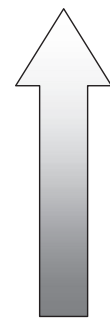

Meta-analysis of RCTs (Highest)

Single large RCT

Cohort study

Case-control study

Case series

Expert opinion

(Lowest)

Fig. 1. Hierarchy of evidence. RCTs: randomized clinical trials.

\section{Avoiding Pitfalls}

\section{Why Is Randomization Important?}

Among single interventional studies, a randomized controlled trial (RCT) is considered to be the highest level of evidence (level 1 evidence). Randomization gives every patient an equal probability of being enrolled to either group. It is the single most important technique to limit bias in clinical research. In a randomized trial of two treatments to improve the healing rate of a fractured hip, the outcome parameter (healing rate) may be influenced not only by the type of treatment that is being investigated, but by numerous other factors (i.e. whether or not the patient smokes, patient's age, severity of the fracture).

The purpose of random allocation of patients in a trial to different treatment groups is to balance known prognostic factors like smoking and patient age, but even more importantly unknown prognostic factors (i.e. those factors the investigators did not realize to be important). Thereby randomization ensures that differences in out-

Table 1. Common pitfalls

\begin{tabular}{|c|c|}
\hline Study design & Common errors \\
\hline Randomized trial & $\begin{array}{l}\text { lack of blinding } \\
\text { lack of concealed randomization } \\
\text { exclusion of dropouts } \\
\text { type II (beta) errors - insufficient sample size } \\
\text { type I (alpha) error - overuse of statistical tests and multiple outcomes }\end{array}$ \\
\hline $\begin{array}{l}\text { Prospective cohort } \\
\text { (with comparison group) }\end{array}$ & $\begin{array}{l}\text { lack of adjustment for differences in characteristics between treatment } \\
\text { and comparison groups } \\
\text { type II (beta) errors - insufficient sample size } \\
\text { type I (alpha) error - overuse of statistical tests and multiple outcomes }\end{array}$ \\
\hline $\begin{array}{l}\text { Prospective case series } \\
\text { (without comparison group) }\end{array}$ & $\begin{array}{l}\text { lack of independent or blinded assessment of outcomes } \\
\text { lack of follow-up }\end{array}$ \\
\hline Case-control study & $\begin{array}{l}\text { recall bias } \\
\text { problems in ascertainment of cases and controls } \\
\text { type II (beta) errors - insufficient sample size } \\
\text { type I (alpha) error - overuse of statistical tests and multiple outcomes }\end{array}$ \\
\hline $\begin{array}{l}\text { Retrospective case series } \\
\text { (with comparison group) }\end{array}$ & $\begin{array}{l}\text { recall bias } \\
\text { type II (beta) errors - insufficent sample size } \\
\text { type I (alpha) error - overuse of statistical tests and multiple outcomes } \\
\text { incomplete reporting in patient charts }\end{array}$ \\
\hline $\begin{array}{l}\text { Retrospective case series } \\
\text { (without comparison group) }\end{array}$ & $\begin{array}{l}\text { incomplete reporting in patient charts } \\
\text { lack of follow-up } \\
\text { recall bias }\end{array}$ \\
\hline
\end{tabular}


come measures mainly reflect the differences in the type of treatment and not the other characteristics of the patient. Whereas other techniques can be used to balance known prognostic factors (e.g. matching, statistical adjustment), randomization is the only technique to control unknown prognostic factors between comparison groups. Thus, studies that fail to randomize patients introduce an important bias in the measurement of the outcome measure.

\section{Why Is Concealment of Randomization Important?}

Equally important is the concept of 'concealment' (not to be confused with blinding). Concealed randomization ensures that surgeons are unable to predict the treatment to which their next patient will be allocated. The safest manner in which to limit this occurrence is a remote 24hour telephone randomization service. Historically, treatment allocations in surgical trials have been placed within envelopes; while seemingly concealed, envelopes are prone to tampering.

Every RCT has exclusion and inclusion criteria, some of which are strict and clear (e.g. patient's age) while others are left to the judgment of the physician (e.g. 'Do you think the patient will be available for a 1-year followup?'). If the physician knows which treatment the patient will receive if the patient is enrolled in the study, he/she might be more or less inclined to enroll the patient in the study based on his/her own preferences and selection bias might occur. It is therefore important that at the time of patient consent and application of the exclusion/inclusion criteria the physician does not know the treatment allocation of the patient. Examples of non-concealed randomization include use of the date (even/odd), hospital chart number, or a randomization schedule that is posted and openly available for viewing. Sealed envelopes, although conventionally thought to be concealed randomization, may not completely conceal allocation. If the envelopes are not opaque, investigators can see the patient allocation without opening the seal and thus could bias the study. If the randomization is concealed, the physician cannot predict to which treatment the patient will be allocated to at the time of patient consent and application of exclusion/inclusion criteria. Randomization techniques that preserve concealment can include telephone and internet-based randomization.

\section{Why Is Blinding Important?}

Blinding also guards against potential bias in a research study. In a study, investigators must ensure that all persons involved do not uncover the treatment alloca- tion of the enrolled patients. In a randomized trial, the patient, the treating physician, the outcome assessor, and the data analyst can be blinded. However, while blinding is feasible in most, if not all, drug trials, it is rarely feasible in a surgical trial. In a surgical trial, operating surgeons can never be blinded but the data analyst can always be blinded. Patients and outcome assessors can sometimes be blinded. Consider a trial comparing surgery versus no surgery for fractures of the wrist. Clearly, blinding of surgeons, patients and outcome assessors is not practical, nor possible, in this study. In a review of orthopedic RCTs, data analysts were never blinded and outcome assessors only $44 \%$ of the time [2].

When blinding is not possible, investigators should take care to ensure independent assessment of the outcome measures if they require some interpretation (i.e. radiographic healing of bone, assessment of clinical outcomes, physical examination). When the outcomes are objective, blinding becomes less necessary (e.g. death or implant breakage).

\section{Why Is an Intention-to-Treat Analysis Important?}

Even when proper randomization is performed, some patients undergo the alternative treatment they were not allocated to either by mistake or the result of technical difficulties with one treatment. The data can be analyzed by performing a per-protocol comparison or an intention-to-treat comparison. In a per-protocol comparison patients that were randomized to treatment $\mathrm{A}$ and indeed received treatment $\mathrm{A}$ are compared to patients that were randomized to treatment $\mathrm{B}$ and received treatment B. For example, patients randomized to receive treatment $\mathrm{A}$ but who actually received treatment B, would then be analyzed in the treatment B group. However, when patients are no longer analyzed in the groups to which they were initially randomized, the balance of prognostic factors achieved through randomization is ruined.

In an intention-to-treat analysis patients are analyzed in the groups to which they were initially randomized. Thus, despite crossing over from treatment A to treatment $\mathrm{B}$, a patient would be treated as if he/she received treatment A. While this approach seems to be counterintuitive at first glance, it preserves the value of randomization: on average known and unknown prognostic factors will be equally distributed across both treatment groups and therefore the treatment effect will be primarily determined by the assigned treatment.

In summary, randomization, concealment of treatment allocation, blinding, and intention-to-treat analysis 


\begin{tabular}{|l|c|c|}
\hline & Truth: positive & Truth: negative \\
\hline Study result positive & True positive & False positive \\
& (1-Beta error) & Alpha error (Type I) \\
\hline Study result negative & False negative & True negative \\
& Beta error (Type II) & (1-Alpha error)
\end{tabular}

Fig. 2. Errors in hypothesis testing.

are all important factors associated with the quality of a study (table 2). The quality of an RCT can be evaluated using several checklists, like the Detsky quality scale [3]. Other scales include the Jadad scale which uses randomization, concealment, double-blinding and assessment of withdrawals and dropouts as key criteria [4].

\section{Errors in Hypothesis Testing}

\section{False-Positive Conclusions in a Study \\ (Alpha Error = Type I Error)}

An alpha error is the erroneous conclusion that there is a difference in groups, when in fact there is none (fig. 2). By convention, most investigators adopt an alpha error rate of 0.05 . Thus, investigators can expect a false-positive result about $5 \%$ of the time $[4,5]$.

The probability of an alpha error increases when the primary outcome parameter has not been stated before the commencement of the trial and comparisons of multiple outcome measures have been performed in the analysis of the trial. The likelihood that some of them will have a $\mathrm{p}<0.05$ and thus the likelihood of an alpha error increases by chance only. Therefore a smaller critical significance level (i.e. smaller alpha level) needs to be established for rejecting the null hypothesis (null hypothesis = no difference between groups) in order to preserve the alpha level for the entire study.

This problem is more prevalent in orthopedic literature than in nonorthopedic surgical and medical literature. In a study of 160 consecutive trials in 15 major journals, Bhandari et al. [6] found that only $33 \%$ of the randomized trials in orthopedic journals provided an explicit statement of the primary outcome parameter compared to $45 \%$ of the trials in nonorthopedic surgical journals and $56 \%$ of the trials in medical journals. Among 40 studies with 182 significant results, only $59 \%$ of the
Table 2. Checklist to avoid pitfalls

1 When comparing two treatments attempt to randomize patients whenever possible

2 Ensure that randomization is concealed

3 Blind anybody in a study that can be blinded i.e. patient, physician, outcome assessor, and data analyst

4 Plan your sample size before the start of the study to avoid false-negative findings (type II error)

5 Ensure that the follow-up of patients is complete

6 If you conduct multiple tests of significance, adjust the $\mathrm{p}$ value to avoid false-positive findings (type I error)

7 Always be cautious of interpreting the results of subgroups within your study; these findings should only be hypothesis generating and not conclusive

results remained significant after statistical adjustment for multiple outcomes.

Alpha errors can be avoided by clearly stating primary and secondary outcome parameters before conducting the trial and adjusting the significance level of secondary outcome measures to the number of calculated secondary outcome parameters, e.g. by using the Bonferroni method $[7,8]$ (alpha $=0.05 / \mathrm{N}$ where $\mathrm{N}=$ number of secondary outcome parameters). For example, with alpha $=0.05$, $\mathrm{p}<0.05$ will be considered statistically significant if only a single outcome measure is analyzed. However, if investigators analyze 10 outcomes the risk of having a falsepositive finding (i.e. $\mathrm{p}<0.05$ ) is approximately $50 \%$ [6]. To guard against this risk of false-positive findings, or alpha error, investigators can correct the final level of statistical significance using the Bonferroni method. Thus, in this example, the new $\mathrm{p}$ value for statistical significance will be $0.05 / 10=0.005$. 
Alternative to the Bonferroni correction, the Yates continuity correlation, O'Brien Fleming group sequential method and the Pocock group sequential method can be used for correction of the significance level for multiple comparisons $[1,5,6]$.

\section{How Do Subgroup Analyses Increase the Risk of} Alpha Error?

Subgroup analyses are frequently post-hoc analyses that risk false-positive results (type I error) in which ineffective (or even harmful) treatments may be deemed beneficial in a subgroup. Conducting multiple statistical tests risks spurious false-positive findings. Alternatively, false-negative results may occur because negative subgroup analyses are often underpowered. Currently, many investigators recommend the cautious interpretation of data derived from an analysis of a subgroup of patients in an RCT.

The problem with subgroup analysis is not restricted to undue emphasis on post-hoc analyses, it extends to a fundamental flaw in how most researchers and clinicians determine if there are differences among subgroups of patients. Most individuals look for differences among subgroups of patients based on separate analyses of treatment effects in each subgroup (i.e. they look for results that are and are not statistically significant among separately analyzed subgroup). However, this approach is fundamentally flawed because within subgroup comparisons cannot inform what we care about (i.e. between subgroup differences). Appropriate techniques such as the Zelen's exact test or the Breslow-Day test can be used to determine if there are qualitative between subgroup differences. Researchers and clinicians should only consider qualitative between subgroup differences (i.e. subgroup interactions) when evaluating subgroups and even then they should be cautious to avoid overemphasizing any differences identified.

Subgroup analyses should be undertaken and interpreted with caution. The validity of a subgroup analysis can be improved by defining a few important (and biologically plausible) subgroups in advance and conducting statistical tests of interaction.

\section{False-Negative Conclusions in a Study}

(Beta Error = Type II Error)

A beta error is the erroneous conclusion that there is no difference between two treatment groups, when in fact there is one (fig. 2). Study power (1 - beta) is the ability of a study to show a difference when one actually exists [10-12]. Typically most investigators adopt a beta error rate of 0.2 or $20 \%$. Thus, investigators can expect a false- negative result about $20 \%$ of the time, which corresponds to a study power of $80 \%$.

Studies with a small sample size are subject to beta errors $[10,11]$. The results of randomized trials may not be convincing when the sample size is not sufficiently large to reveal a true difference between treatment groups. Many investigators agree that beta error rates of $>20 \%$ (study power of $<80 \%$ ) are subject to an unacceptably high risk of false-negative results $[5,13,14]$.

Lochner et al. [15] investigated beta errors of 117 randomized trials in literature dealing with orthopedic trauma. The mean overall study power among all trials was $25 \% .106$ of 117 trials $(91 \%)$ had a beta $>0.2$ (= power $<80 \%$ ) for the primary outcome.

Underpowered studies have ethical implications. They have limited value in providing meaningful clinical data for evidence-based medicine while participants are misled that their enrollment will benefit future patients. For example if a study comparing an operative versus nonoperative treatment shows no difference, most patients will choose nonoperative treatments. However, if this conclusion is wrong (i.e. falsely negative) and the operative treatment actually has an advantage that could not have been detected due to insufficient study power, the conclusion of the study may justify inferior treatment.

Beta errors can be avoided by an a priori power and sample size calculation and a realistic assessment of the feasibility of a study, considering incidence of the investigated problem, enrollment time, single versus multicenter approach and financial resources.

\section{How Can Meta-Analysis Reduce Beta Error?}

In order to overcome the sample size limitations of a single RCT or to increase the precision of a treatment effect, meta-analyses are frequently used in medical literature.

Meta-analyses are systematic reviews that summarize and combine the results of multiple studies, typically RCTs, in order to answer a focused clinical question. By combining the results of multiple RCTs the sample size increases and smaller differences between treatment groups can be detected. False-negative results are therefore less likely; for example, multiple small RCTs have investigated whether treatment A or treatment $\mathrm{B}$ results in a lower complication rate for a certain condition/disease. Seven RCTs with an average sample size of 50 patients found no significant differences in complication rates between treatments $\mathrm{A}$ and $\mathrm{B}$. However, each study is limited by the small sample size. It is not clear, whether there is truly no difference in com- 
plication rates between both treatments or the studies lack statistical power to detect a difference. If the results of these studies are combined using meta-analytic techniques, the total size becomes 350 patients and statistical power to detect smaller difference between treatment groups becomes greater. One such example of the value of meta-analysis comes from the surgical literature evaluating two techniques (reamed versus unreamed intramedullary nailing) in the operative treatment of tibial shaft fractures. All of nine small RCTs did not identify any significant difference between groups; however a subsequent meta-analysis of these small RCTs had sufficient study power to identify a difference in reduction of the risk of reoperation with reamed nailing.

Meta-analyses are limited by the heterogeneity of the pooled studies. Heterogeneity relates to differences in the individual studies with regard to the studied population, study design, study conduction, data analysis or any other known or unknown factors. It is therefore paramount to assess the heterogeneity before pooling results. Heterogeneity can be assessed mathematically using the confidence interval of the outcome measure. Nonoverlapping 95\% confidence intervals indicate great underlying differences between studies. If the studies are too heterogeneous the results should not be pooled.

\section{How Can the Required Sample Size of a Study Be Calculated?}

Calculating the appropriate sample size can be a challenging task for many investigators. A sample size calculation before the commencement of a trial is important (a priori) to estimate an appropriate sample size and to avoid false-negative results. In addition, funding bodies and ethics committees often require a sample size calculation and justification for the sample size from investigators prior to approving the study. Moreover, a careful sample size determination often infers increasing expertise of the investigative team and improves chances of funding. Several free sample size calculators are available on the internet (i.e. http://biostat.mc.vanderbilt.edu/twiki/bin/view/Main/PowerSampleSize).

The magnitude of the difference in the primary outcome parameter that the investigators consider clinically relevant should be the basis for the sample size calculation, (i.e. union rates) when comparing two different treatment methods for fracture fixation. A significance level of $\mathrm{p}=0.05$ (alpha $=0.05)$ is used by convention. This means that investigators accept a $5 \%$ chance of an alpha error (false-positive). The sample size calculation will reveal how many patients per group are necessary to show a real difference, if one exists.

In addition to the hypothesized difference in the primary outcome parameter and the significance level (usually alpha $=0.05$ ), the acceptable power of the study, and the anticipated standard deviations of the primary outcome parameter in the two groups need to be established before proceeding with the sample size calculation. A study power of 0.8 is a conventionally accepted standard, which means that the investigators are willing to accept a $20 \%$ probability that there is no difference between two groups when a difference actually exists (beta error). Any increase in study power and decrease of the alpha level of significance will result in a higher sample size requirement. The anticipated standard deviations in the two groups can be either determined by performing a preliminary pilot study or from data in the literature. If no data are available, they can only be estimated.

Sample sizes can be calculated manually or a computer program can be used. Numerous software packages exist for sample size determination. The author's preferred software is InStat-StatMate (GraphPad Software Inc., San Diego, Calif., USA). If no software is available, the following formula can be used in order to determine sample size:

(a) Comparing two means (continuous variable)

$$
\mathrm{n}_{1}=\mathrm{n}_{2}=\left(\sigma_{1}{ }^{2}+\sigma_{2}{ }^{2}\right)\left(\mathrm{z}_{1-\alpha / 2}+\mathrm{z}_{1-\beta}\right)^{2} / \Delta^{2}
$$

where $\mathrm{n}_{1}=$ sample size of group one, $\mathrm{n}_{2}=$ sample size of group two, $\alpha=$ significance level alpha (usually 0.05), $\beta=$ 1-power (beta/usually 0.2), $\Delta=$ difference $=\mu_{2}-\mu_{1}, \mu_{1}$, $\mu_{2}=$ sample means, $\sigma_{1}, \sigma_{2}=$ sample standard deviations.

(b) Comparing binomial proportions (percentages for dichotomous variables)

$$
n_{1}=n_{2}=\left[\left(2 p_{m} q_{m}\right)^{1 / 2} z_{1-\alpha / 2}+\left(p_{1} q_{1}+p_{2} q_{2}\right)^{1 / 2} z_{1}-\beta\right]^{2} / \Delta^{2}
$$

where $\mathrm{n}_{1}=$ sample size of group one, $\mathrm{n}_{2}=$ sample size of group two, $\alpha=$ significance level alpha (usually 0.05), $\beta=$ 1-power (beta/usually 0.2 ), $\mathrm{p}_{1}, \mathrm{p}_{2}=$ sample probabilities, $\mathrm{q}_{1}, \mathrm{q}_{2}=1-\mathrm{p}_{1}, 1-\mathrm{p}_{2}, \mathrm{p}_{\mathrm{m}}=\left(\mathrm{p}_{1}+\mathrm{p}_{2}\right) / 2, \mathrm{q}_{\mathrm{m}}=1-\mathrm{p}_{\mathrm{m}}, \Delta=$ difference $=p_{2}-p_{1}$. 


\section{What Are the Important Elements of a Study Protocol?}

When developing a study protocol typically an abstract, an overview of the research plan, a budget and justification, resources and environment and overall considerations need to be included (table 3 ).

The abstract is an important part of a study protocol because it is the first page that a reviewer reads. Reviewers of granting agencies may make up their opinion based on the abstract alone. It may be difficult to overcome a bad first impression and conversely there may be a lot to gain with a good first impression. The purpose of the abstract is to describe succinctly every key element of the proposed project. It is good to be complete, but concise.

The purpose of the research plan is to describe what will be done, why it is important and how the study will be conducted. The main elements of the research plan are the specific aims of the study, the background of the topic and significance of the problem, and research design and methodology. Prior work relevant to the proposed project should be included. Also if a pilot study was conducted, the results should be included. The purpose of the specific study aims is to clearly describe what research question the investigators are trying to answer by conducting the study. In detail, the hypothesis of the study and the long-term goals should be stated. The purpose of the background and significance section is to lay out the rationale for the proposed research project and to summarize currently available data in literature that is relevant to the project. The research design and the methodology used in the process of planning and conducting the project should be described in detail.

All elements of the study protocol can be crucial to how favorably an application is reviewed. It is important to convince granting agencies that the research team has the required know-how and the environmental resources to conduct the study. It can be helpful to include the professional background of the investigators (clinicians, trialists, statisticians, etc.) and eventually their track record with similar projects. Available environmental resources (e.g. lab space) required to conduct the study should be listed.

The purpose of the budget and justification is to present all expenses required to achieve the project aims. The budget can include personnel, consultants, equipment, supplies, travel and any other expenses. However, it is important to be realistic and justify all expenses.

Pitfalls in Clinical Research
Table 3. Guide for randomized trial protocols (Canadian Institutes of Health Research)

Part 1 The need for the trial

1 What is the problem to be addressed?

2 What are the principal research questions to be addressed?

3 Why is a trial needed now?

4 Give references to any relevant systematic reviews and discuss the need for your trial in light of these reviews

5 How will the results of this trial be used?

6 Describe any risks to the safety of the participants involved in this trial

Part 2 The proposed trial

1 What is the proposed trial design?

2 What are the proposed trial interventions?

3 What are the proposed practical arrangements for allocating participants to trial groups?

4 What are the proposed methods for protecting against sources of bias?

5 What are the planned inclusion/exclusion criteria?

6 What is the proposed duration of the treatment period?

7 What is the proposed frequency and duration of follow-up?

8 What are the proposed outcome measures?

9 How will the outcomes be measured at follow-up?

10 Will health services questions be measured at follow-up?

11 What is the proposed sample size and what are the justifications for the assumptions underlying the power calculations?

12 What is the planned recruitment rate?

13 Are there likely to be problems with compliance?

14 What is the likely rate of loss to follow-up?

15 What is the proposed type of analyses?

16 What is the proposed frequency of analyses?

17 Are there any planned subgroup analyses?

18 Has a pilot study been carried out using this design?

Part 3 Trial management

1 What are the arrangements for day-to-day management of the trial?

2 What will be the role of each investigator or collaborator in this trial?

3 Describe any committees such as the steering committee or data monitoring and safety committee (if applicable)

Different granting agencies may have different requirements for the study protocol, but typically they have the above mentioned elements in common. However, the length and detailed structure needs to be adjusted to the guidelines of the targeted granting agency. 


\section{Summary}

With the ever-increasing amount of available information, clinicians must consider a shift in paradigm from traditional practice to one that involves question formulation, validity assessment of available studies and appropriate application of research evidence to individual patients. Key considerations in identifying the pitfalls in a published clinical research study include study design, errors in hypothesis-testing, appropriate sample size considerations. A basic understanding of these principles of research will empower both investigators and readers when applying the results of research to clinical practice.

\section{References}

1 Sackett DL, Richardso WS, Rosenberg WM: Evidence Based Medicine: How to Practice and Teach EBM. New York, Churchill Livingstone, 1997.

-2 Bhandari M, Richards RR, Sprague S, Schemitsch EH: The quality of reporting of randomized trials in the Journal of Bone and Joint Surgery from 1988 through 2000. J Bone Joint Surg Am 2002;84-A:388-396.

$\checkmark 3$ Detsky AS, Naylor CD, O'Rourke K, McGeer AJ, L'Abbe KA: Incorporating variations in the quality of individual randomized trials into meta-analysis. J Clin Epidemiol 1992;45:255265.

-4 Jadad AR, Moore RA, Carroll D, Jenkinson C, Reynolds DJ, Gavaghan DJ, McQuay HJ: Assessing the quality of reports of randomized clinical trials: is blinding necessary? Control Clin Trials 1996;17:1-12.
75 Streiner DL: Sample size and power in psychiatric research. Can J Psychiatry 1990;35:616620 .

6 Dorrey F, Swiontkowski MF: Statistical tests. What they tell us and what they don't. Advances Orthop Surg 1997;21:81-85.

7 Bhandari M, Whang W, Kuo JC, Devereaux PJ, Sprague S, Tornetta P 3rd: The risk of falsepositive results in orthopaedic surgical trials Clin Orthop 2003:63-69.

$>8$ Cupples LA, Heeren T, Schatzkin A, Colton T: Multiple testing of hypotheses in comparing two groups. Ann Intern Med 1984; 100:122129.

$>9$ Senghas RE: Statistics in the journal of bone and joint surgery: suggestions for authors. J Bone Joint Surg Am 1992;74:319-320.

10 Guyatt G, Jaeschke R, Heddle N, Cook D, Shannon H, Walter S: Basic statistics for clinicians. 1. Hypothesis testing. CMAJ 1995;152: 27-32.
11 Guyatt G, Jaeschke R, Heddle N, Cook D, Shannon H, Walter S: Basic statistics for clinicians: 2 . Interpreting study results: confidence intervals. Can Med Assoc J 1995;152:169_ 173.

12 Chung KC, Kalliainen LK, Hayward RA: Type II (beta) errors in the hand literature: the importance of power. J Hand Surg [Am] 1998;23: 20-25.

13 Staquet MJ, Rozencweig M, Von Hoff DD, Muggia FM: The delta and epsilon errors in the assessment of cancer clinical trials. Cancer Treat Rep 1979;63:1917-1921.

14 Williams JL, Hathaway CA, Kloster KL, Layne $\mathrm{BH}$ : Low power, type II errors, and other statistical problems in recent cardiovascular research. Am J Physiol 1997;273:H487-H493.

15 Lochner HV, Bhandari M, Tornetta P, 3rd: Type-II error rates (beta errors) of randomized trials in orthopaedic trauma. J Bone Joint Surg Am 2001;83-A:1650-1655. 